Investigación

\title{
VIVIR CON DOLOR CRÓNICO DESDE LA EXPERIENCIA DE ADULTOS MAYORES CON ENFERMEDADES CRÓNICO DEGENERATIVAS
}

\section{LIVING WITH CRONIC PAIN FROM THE EXPERIENCE OF OLDER ADULTS WITH CHRONIC DEGENERATIVE DISEASES}

\section{Katia Ruiz-Aguilar}

Estudiante de pregrado. Centro Universitario Siglo XXI, México

katia.ruiz@cusigloxxi.edu.mx

http://orcid.org/0000-0002-4081-3263.

\section{Vidal Chan-Castro}

Estudiante de pregrado. Centro Universitario Siglo XXI, México

vidal.chan@cusigloxxi.edu.mx

http://orcid.org/0000-0002-4836-2460

\section{Gabriela Hernández-Gómez}

Estudiante de pregrado. Centro Universitario Siglo XXI, México gabriela.hernandez@cusigloxxi.edu.mx

http://orcid.org/0000-0002-8466-7990

\section{Alan Cab-López}

Estudiante de pregrado. Centro Universitario Siglo XXI, México

alan.cab@cusigloxxi.edu.mx

http://orcid.org/0000-0003-1484-3415

\section{Janet Espadas-Uuh}

Estudiante de pregrado. Centro Universitario Siglo XXI, México

janet.espadas@cusigloxxi.edu.mx

http://orcid.org/0000-0001-8190-8077

\section{Josué Medina-Fernández}

Licenciado en Enfermería y Maestro en Enfermería. División Ciencias de la Salud de la Universidad de Quintana Roo, México

josuemedinafernandez@outlook.es

http://orcid.org/0000-0003-0588-9382

Artículo recibido el 4 de agosto de 2021. Aceptado en versión corregida el 29 de noviembre de 2021 . 
Vivir con dolor crónico desde la experiencia de adultos mayores...

\title{
RESUMEN
}

INTRODUCCIÓN: Ser adulto mayor conlleva a cambios físicos, psicológicos y sociales que se ven aún más afectados por comorbilidades como las enfermedades crónicas y el dolor. El propósito es interpretar las experiencias de vivir con dolor de los adultos mayores con enfermedades crónico-degenerativas. METODOLOGÍA: Diseño cualitativo de tipo fenomenológico, recolectado mediante un muestreo intencional a través de una entrevista semi estructurada, en la cual se seleccionó a la población adulta mayor con una patología crónico-degenerativa que haya experimentado dolor crónico; se analizaron los datos con el proceso cognitivo de Janice Morse, hasta llegar a la saturación de la información. Participaron 8 personas entre los 66 y 72 años, habiendo siete mujeres y un hombre. RESULTADOS: Se encontraron cuatro categorías: 1. El desgaste de vivir con dolor; 2. Cotidianidad del vivir con dolor; 3. Alternativas para aliviar el dolor, y 4. Afectación en el entorno social. CONCLUSIÓN: Ser adulto mayor trae muchos cambios en todas las esferas de la vida, sin embargo, el padecer una enfermedad crónica acelera toda esta transición, viéndose afectados la salud mental, físico y social, adaptando esta situación a su cotidianidad, buscando a su vez medios alternos que mitiguen o disminuya el dolor.

Palabras clave: Acontecimientos que cambian la vida, dolor crónico, adulto mayor.

\begin{abstract}
INTRODUCTION: Being an older adult leads to physical, psychological, and social changes that are further affected by comorbidities such as chronic diseases and pain. The purpose is to interpret the experiences of living in pain of older adults with chronic-degenerative diseases. METHOD: Qualitative phenomenological research, recollected by an intentional sampling through a semi-structured interview in which was selected the adult population with a chronic-degenerative pathology that have experienced an state of chronic pain. The data was analyzed with the cognitive process of Janice Morse and reached to a saturation of 8 participants. There were 8 participants between the ages of 66 and 72, with seven women and one man. RESULTS: Four categories were found: 1. Wearing of living with pain; 2. Daily Living with Pain; 3. Alternatives for Pain Relief, and 4. Social Affectation. CONCLUSION: Being an older adult brings many changes in all spheres of life, however, suffering from a chronic disease accelerates this whole transition, being affected mental, physical and social health, adapting this situation to its daily life, seeking in turn alternate means to mitigate or decrease pain.
\end{abstract}

Keywords: Life-changing events, chronic pain, older adults.

\section{http://dx.doi.org/10.7764/Horiz_Enferm.32.3.297-305}

\section{INTRODUCCIÓN}

El vivir con dolor ha causado un impacto en los Adultos Mayores (AM), quienes pa- decen este síntoma, ya que suele afectar diferentes aspectos personales en la vida 
Ruiz-Aguilar K, Chan-Castro V, Hernández-Gómez G, Cab-López A, Espadas-Uuh J, Medina-Fernández J

de cada individuo, como salud, autonomía, independencia, satisfacción con la vida, y aspectos ambientales como redes de apoyo, servicios sociales, entre otros ${ }^{(1)}$.

Actualmente la edad de vida de una persona supera los 60 años, por lo que, en México, se considera AM a aquella persona que tiene más de 60 años y se refiere a la etapa que suma todas las experiencias de la vida y que ha pasado por la mayoría de las metas familiares, profesionales y sociales ${ }^{(1)}$. Desde el 2015 al 2020 el aumento de esta población es significante; con base en la Encuesta Nacional de la Dinámica Demográfica (ENADID) 2018, en el país residen 15,4 millones de personas de 60 años o más, de las cuales 1,7 millones viven solas ${ }^{(2)}$.

Las enfermedades crónico-degenerativas son patologías de larga duración, cuya evolución es generalmente lenta y representan una verdadera epidemia que va en aumento, debido al envejecimiento de la población y los estilo de vida actual que acentúan el sedentarismo y la inadecuada alimentación; cuando estas enfermedades se detectan en etapas avanzadas o bien, no llevan un control establecido, suelen generar diversos síntomas muy molestos, y hasta perjudiciales para la salud del individuo, siendo las manifestaciones transversales característico en muchas de las patologías crónicas.

De esta manera, el dolor crónico se define como un síntoma cuya duración se extiende por más de tres meses desde que remite la lesión que lo generó, se repite y se asocia a enfermedades crónicas que aumentan con la edad, se localiza frecuentemente en las principales articulaciones, espalda, piernas y pies $^{(3)}$. Existe una separación entre el dolor asociado a la enfermedad y la queja de dolor, pudiendo provoca déficit en los aspectos físicos, psicológicos y sociales.

Dicho lo anterior, enfermería cumple un papel único, ya que facilita la adaptación al analizar el comportamiento del AM, además de mejorar las interacciones del entorno enfocado a la experiencias del dolor en este grupo vulnerable, pues las estrategias de enfermería normalmente se centran en la medición y alivio del dolor; sin embargo, debemos enfocarnos en aquellos factores psicológicos, sociales y espirituales que pueden ayudar a sobrellevar esta afectación, por lo que primero se requiere abordar esos factores cualitativamente y así poder brindar un plan de cuidados adaptado al AM con dolor crónico. Con base a lo anteriormente mencionado se platea el siguiente propósito: Interpretar las experiencias de vivir con dolor de los adultos mayores con enfermedades crónico-degenerativas.

\section{METODOLOGÍA}

Se realizó un estudio cualitativo de tipo fenomenológico-descriptivo sobre las experiencias de vivir con dolor crónico en adultos mayores con enfermedades crónicas degenerativas. En el estudio participó una muestra de ocho personas pertenecientes a la península deYucatán, recolectado a través de un muestreo intencional mediante una entrevista semi estructurada. Entre los criterios de inclusión fueron adultos mayores que viven con una patología crónica que cause dolor con diagnóstico médico confirmado, haber tenido una calificación igual o mayor de 9 en la prueba Pffeifer (que mide deterioro cognitivo) y que haya firmado el 
Vivir con dolor crónico desde la experiencia de adultos mayores...

consentimiento informado. Durante la recopilación de datos se tomaron en cuenta las consideraciones éticas, cumpliendo lo establecido en el reglamento de la Ley General de Salud en materia de investigación, aplicando el artículo 13 del capítulo 1, título segundo, tratando con respeto y protegiendo su bienestar, aunado a que se cumple con los requisitos de acuerdo al código de Helsinky.

El plan de recolección se llevó a cabo entregando previamente el consentimiento informado, seguido de la autorización para poder grabar las entrevistas. Posterior ala entrevista y saturación de datos, se analizó con el proceso cognitivo de Janice Morse, cuyo proceso consiste en comprender, sintetizar, teorizar y recontextualizar.

\section{Comprender}

Se inició desde la ubicación de los participantes en cumplimiento de los criterios de la investigación (ser adulto mayor, haber sido diagnosticado con alguna enfermedad crónica de al menos 5 años, tener una puntuación de 9 y 10 puntos con la escala Pfeiffer que mide probable deterioro cognitivo y haber tenido al menos en la última semana una escala EVA de 6 puntos), la identificación del diálogo por medio de las preguntas detonadoras y regeneradoras. Del resultado del diálogo se obtuvieron los datos que nos permitirán describir lo expresado por los participantes.

\section{Sintetizar}

Es la relación entre la descripción y la transcripción de forma literal por los investigadores, haciendo una lectura minuciosa de la entrevista transcrita e identificado ideas y argumentos relevantes expresados por los participantes, mediante una codificación de color, llevando un orden de los argumentos en común.

\section{Teorizar}

Se interpretaron los significados, se extrajeron las conclusiones que guiaron la investigación y se desarrolló a partir de la comprensión y síntesis de los datos. La teoría brindó una estructura mediante la interpretación de los datos y fueron definidos conceptualmente mediante un orden de lo más referido a lo menos referido.

\section{Recontextualización}

Seguidamente, los resultados fueron colocados en el contexto del conocimiento establecido para poder identificar claramente que apoyan a la explicación, estableciendo una base de conceptos bien definidos vinculándose con los conocímientos de la ciencia, así como con la filosofía de Heidegger. Se aplicó una triangulación de datos, la cual consistió en regresar con los entrevistados para confirmar que la interpretación realizada es lo señalado por los participantes; otra triangulación que se llevó a cabo es la del investigador, teniendo como fin analizar el discurso desde diversos puntos de vista de los investigadores con el objetivo de atribuir un verdadero significado al fenómeno y por último la triangulación espacial, donde los participantes deberán de ser de diversos puntos de la comunidad para observar si la percepción del fenómeno son similares. 
Ruiz-Aguilar K, Chan-Castro V, Hernández-Gómez G, Cab-López A, Espadas-Uuh J, Medina-Fernández J

\section{RESULTADOS}

Participaron ocho adultos mayores con un rango de edad entre los 66 y 72 años, siendo siete mujeres y un hombre pertenecientes a la península de Yucatán. De acuerdo con el estado civil, dos son viudas, cuatro casados y dos divorciados, de los cuales tres personas viven solas y cinco acompañadas de un familiar. Entre las enfermedades crónicas que padecen los participantes, tres padecen artritis, tres diabetes y dos fibromialgias, oscilando entre 5 y 10 años de vivir con el dolor. Se encontraron cuatro categorías, lo que se presenta a continuación:

\section{El desgaste de vivir con dolor}

Los participantes lo refieren como un sentimiento intenso de tristeza, derrota del cuerpo y de la mente asociada a una experiencia sensorial, familiar y social.

P7(67 años)- "es horrible, es una cosa muy horrible, porque no es vivir, es sobrevivir, porque la verdad no estoy tranquila, no vivo feliz desde hace mucho tiempo, desde que nací creo he estado sufriendo..."

\section{Cotidianidad del vivir con dolor}

Los participantes lo refieren como una limitante para la realización de las actividades cotidiana que requieren de esfuerzo físico, siendo un impedimento para ellos toda acción, ya sea desde la más básica hasta la más compleja, el cual afecta directamente en la libertad de movimiento y el traslado, por lo que éstos recurren a ocupar dispositivos de apoyo y la ayuda de una persona.

P4 (66 años) - "lo mucho que puedo caminar son de 10 a 20 metros y me empieza a doler la rodilla y a veces ya ni me puedo levantar de la silla, porque no puedo flexionar bien la rodilla, $y$, pues me quedo sentado o mi esposa me ayuda para que me lleve a acostarme a la hamaca, y si ella no está, me ayudo con mi bastón o me agarro de las paredes...la enfermedad me está consumiendo poco a poco..."

\section{Alternativas para aliviar el dolor} Los participantes lo refieren como el uso de medicina complementaria para disminuir el dolor, siendo de gran ayuda para ellos como una parte complementaria de su tratamiento, debido a que suelen sentirse menos dependientes del medicamento, aunado a que son creencias que se les ha enseñado como un método para utilizarlos en ellos mismos a lo largo de su vida.

\section{P5 (67 años) "pues trato de} dormir, a veces he tomado mis té, así cositas para que se me calmen mi dolor, o trato de salir a la puerta de mi casa, distraer la mente y estar tranquila, al menos para ocupar mi mente..."

\section{Afectación del entorno social}

Los participantes lo refieren como un aspecto negativo en su vida, ya que se sienten aisladas del ambiente que los rodea, siendo parte de su decisión individual o porque las circunstancias como el dolor o alguna condición física, no se los permite, viéndose afectados sus redes sociales como la familia y los amigos.

P8 (67 años) "ya no es lo mismo de antes, la relación con familia y amigos cambió, me siento cansada, me aíslo...prefiero estar acostada y sola..."

\section{DISCUSIÓN}

En el estudio participaron ocho personas entre los 62 años y 72 años, habiendo siete 
Vivir con dolor crónico desde la experiencia de adultos mayores...

mujeres y un hombre. Esto se justifica, ya que de acuerdo con ENSANUT (2018) se observó en México un incremento de la prevalencia de enfermedades crónicas conforme aumenta la edad, siendo el grupo de 60 años y más el de la mayor prevalencia $^{(4)}$.

Las diferencias entre una persona y otra en la disminución de las funciones cognitivas asociadas a la edad dependen de muchos factores, como la situación socioeconómica, el estilo de vida, la presencia de enfermedades crónicas ${ }^{(5)}$. Algunas revisiones indican que, en personas de mayor edad, hay disminución en los niveles de endorfinas y encefalinas, mientras que otros expertos tienen la hipótesis que dicha reducción en los niveles de estos opioides endógenos y la posible reducción en los niveles de transmisores en las vías inhibitorias, pueden producir un aumento en la percepción del dolor en las personas mayores con enfermedades crónicas ${ }^{(6)}$.

En la categoría uno, llamado el desgaste de vivir con dolor, hace referencia al deterioro físico derivado del proceso natural del envejecimiento, asociado a la presencia de una o más enfermedades y al estilo de vida del individuo, puede provocar que los adultos mayores no consigan realizar por sí mismos sus actividades, debido al desgaste tanto físico como mental.

Las autoras Alvarado y Salazar llevan a concluir que el dolor afecta a todos los aspectos de la vida, y que los adultos mayores utilizan la capacidad de recuperacióny reconocen el valor de tener una actitud positiva, aceptando y aprendiendo a vivir con el dolor ${ }^{(7)}$.
De igual forma nos comparten que los adultos mayores experimentan una serie de sentimientos y emociones como la angustia, la tristeza, el miedo, el estrés, la impotencia, la desesperanza y el aislamiento, los cuales dan cuenta de una nueva perspectiva que lleva a entender aún más cómo la experiencia del dolor crónico impacta en todas las dimensiones de los participantes, esta mezcla de emociones sugiere posterior desgaste físico y mental en el adulto mayor, despojándolo de toda su energía y arrastrándolo a experimentar emociones negativas que agudizarán sus síntomas y afectarán a otras funciones normales de su cuerpo $^{(8)}$. En esta primera categoría nos podemos dar cuenta que los procesos fisiológicos en el AM resultan con base al deterioro de su enfermedad crónica, disminuyendo la funcionalidad, volviéndose más vulnerables a las enfermedades y al desgaste físico.

La segunda categoría, llamada cotidianidad del vivir con dolor, se observa que se genera con las enfermedades crónicas un gran impacto en la calidad de vida y en el estado funcional que incluyen limitación en movimientos y trastornos del equilibrio. Arango y Ruiz resaltan que, para el adulto mayor, hablar en términos de calidad de vida, suele estar vinculado al mantenimiento de la funcionalidad, cuyos componentes principales son, la independencia y la autonomía ${ }^{(9)}$. Teniendo en cuenta a Silva y Jiménez, señalan que el hecho de presentar dependencia frente a alguna situación ocasiona mayor vulnerabilidad, disminuyendo el bienestar y la calidad de vida ${ }^{(10,11)}$.

La tercera categoría, se llama alternativas para mitigar el dolor, la cual 
refiere como una alternativa para aliviar o mitigar el dolor, también es abordado por Gómez y Medina, el cual nos refieren que actualmente el dolor físico se ha convertido en un problema que puede ser resuelto acudiendo una buena medicina hegemónica, tradición o a las diferentes técnicas contra el dolor ${ }^{(13)}$.

Alvarado y Salazar refieren que los adultos mayores, al entender que el dolor es parte de su vida, la aceptación y las estrategias de adaptación son para hacer frente al difícil proceso de manejarlo; para el cual se desarrollan una serie de estrategias creativas con base a prueba y error y compartiendo experiencias con otras personas que experimentan sitúaciones similares, con el espíritu de la búsqueda de una nueva vía para aliviar su dolor $^{(8)}$.

La cuarta categoría, de nombre afectación del entorno social, ha surgido a partir de lo referido por parte de los participantes, ya que ellos mencionan cuáles son las medidas terapéuticas para manejar su dolor, destacando que, además del uso de los medicamentos, ellos acuden a otro tipo de remedio, ayuda, alternativa para poder sobrellevar el dolor, y eso es muy benéfico a nivel físico, ya que en muchas ocasiones la alternativa suele tener alivio en la región o regiones de dolor, y también a nivel psicológico, debido a que el individuo siente un confort al acudir con un recomendado o bien, haciendo uso de sus creencias inculcadas en su niñez, dependiendo de la cultura de éste haya tenido. Dicho lo anterior, el dolor modifica no solo el aspecto físico, sino que también el aspecto social, debido a las experiencias vividas, a la capacidad de resiliencia y los factores socioculturales del $\mathrm{AM}^{(14-16)}$.
Se recomienda en esta investigación darle un seguimiento e intervención desde el punto de vista enfermero y médico que coadyuve a mejorar la salud mental, física y el aspecto social.

\section{CONCLUSIÓN}

Ser adulto mayor trae muchos cambios en todas las esferas de la vida, sin embargo, el padecer una enfermedad crónica acelera toda esta transición, viéndose afectados la salud mental, físico y social, adaptándose esta situación a su cotidianidad, buscando a su vez medios alternos que mitiguen o disminuya el dolor.

Es muy importante identificar cómo los adultos mayores describen sus vivencias al dolor, permitiendo una mejora de los cuidados de enfermería adaptando a su situación actual y a su patología.

\section{REFERENCIAS BIBLIOGRÁFICAS}

1. PENSIONISSSTE. Día del adulto mayor [Internet]. gobierno de México. $2017 . \quad$ Disponible en: https://www.gob.mx/pensionissste/arti culos/dia-del- adulto-mayor123010?idiom=es

2. Instituto Nacional de Estadística y Geografía [INEGI]. Estadísticas a Propósito Del Día Internacional De Las Personas De Edad. Inst Nacional de Estadística y Geografía [Internet]. 2019;1-9. Disponible en: https://www.inegi.org.mx/contenidos/s aladeprensa/aproposito/2019/edad201 9_Nal.pdf

3. Gil Gregorio P, Fernández Alonso C, Gutiérrez Rodríguez J, López Mongil R, López Trigo JA, Viloria Jiménez 
Vivir con dolor crónico desde la experiencia de adultos mayores...

MA. Guía de la buena práctica clínica en geriatría. Dolor crónico en el anciano. Atención primaria de calidad. 2013. 1-120 p.

4. Shamah-Levy T, Vielma-Orozco E, Heredia-Hernández O, RomeroMartínez M, Mojica-Cuevas J, CuevasNasu L, et al. Encuesta Nacional de Salud y Nutrición 2018-19 Resultados nacionales [Internet]. Vol. 53, Instituto Nacional de Salud Pública. 2020. 1689-1699 p. Disponible en: https://ensanut.insp.mx/encuestas/ensa nut2018/doctos/informes/ensanut_20 18_informe_final.pdf

5. Instituto Nacional de Estadística y Geografía [INEGI]. Encuesta Nacional sobre Salud y Envejecimiento en México 2018. Disponible en: https://ensanut.insp.mx/encuestas/ensa nut2018/doctos/informes/ensanut_201 8_informe_final.pdf

6. Medina Fernández IA, Medina Fernández JA, Cervera Baas ME, Gallegos Torres RM, Casco Gallardo KI, Carrillo Cervantes AL. Desempeño físico y condiciones crónicas padecidas en adultos mayores. [Internet]. $15 \mathrm{de}$ abril de 2020 [citado 4 julio de agosto de 2021];30(3):221-3. Disponible en: http://horizonteenfermeria.uc.cl/index. php/RHE/article/view/10716

7. Alvarado-García AM, Salazar-Maya AM. Descubriendo los sentimientos y comportamientos que experimenta el adulto mayor con dolor crónico benigno. Gerokomos. 2016;27(4):1426.

8. Alvarado-García AM, Salazar-Maya ÁM. Aprender a vivir con el dolor crónico en la vejez. Salud UNINORTE. 2018;34(2):349-62.
9. Arango VE, Ruiz IC. Diagnóstico de los Adultos Mayores en Colombia. Fund Saldarriaga Concha [Internet]. 2005;184. Disponible en: http://medcontent.metapress.com/inde x/A65RM03P4874243N.pdf\%5Cnhttp: I

/www.dane.gov.co/files/comunicados/ cp_informfinal08.pdf

10. Silva J, Coelho S, Pereira T, Marques S. Caídas en el adulto mayor y su relación con la capacidad funcional. Rev Lat Am Enfermagem. 2012;20(5):1-9.

11. Pedro Enrique Jiménez, Fidel LópezEspuela, Juan Carlos Portilla-Cuenca, Juan Diego Pedrera-Zamorano, M. Antonia Jiménez-Gracia, Jesús $\mathbf{M}$. Lavado-García IC-N, Introducción. Valoración de las actividades instrumentales de la vida diaria tras un ictus mediante la escala de Lawton y Brody Pedro. Phys Ther. 2013;93(5):681-93.

12. Flores G, Rivas R, Seguel P. Nivel De Sobrecarga En El Desempeño Del Rol Del Cuidador Familiar De Adulto Mayor Con Dependencia Severa. Cienc y enfermería. 2012;18(1):29-41.

13. Verónica H., Iniestra K, Martínez, P. Sufrimiento en el adulto mayor: causas y soluciones. un análisis por sexo [Tesis de Licenciatura]. Estado de México: Universidad Autónoma del Estado de México;2015. Recuperado de: http://ri.uaemex.mx/handle/20.500.117 99/66619

14. Francisco L. Definición y clasificación del dolor. Diaz. 1985;130(2):49-51.

15. Organización Mundial de la Salud. Envejecimiento activo: un marco político. 2002;37:74-105.

16. Bragard CD. Evaluación del dolor: 
Ruiz-Aguilar K, Chan-Castro V, Hernández-Gómez G, Cab-López A, Espadas-Uuh J, Medina-Fernández J

aspectos metodológicos y uso clínico.

Disponible

en:

EMC - Kinesiterapia - Medicina https://www.sciencedirect.com/science Física [Internet]. 2010; 31(4):1-11. /article/abs/pii/S1293296510707155\#! 